\title{
Molecular Assembly in Block Copolymer-Surfactant Nanoparticle Dispersions: Information on Molecular Exchange and Apparent Solubility from High-Resolution and PFG NMR
}

\author{
Guilherme A. Ferreira ${ }^{1, *}++^{\mathbb{D}}$, Watson Loh ${ }^{1}\left(\mathbb{D}\right.$, Daniel Topgaard ${ }^{2} \mathbb{D}$, Olle Söderman ${ }^{2}$ and Lennart Piculell $^{2, *}$ \\ 1 Institute of Chemistry, University of Campinas (UNICAMP), P.O. Box 6154, Campinas 13083-970, Brazil; \\ wloh@unicamp.br \\ 2 Division of Physical Chemistry, Lund University, P.O. Box 124, 22100 Lund, Sweden; \\ daniel.topgaard@fkem1.lu.se (D.T.); olle.soderman@fkem1.lu.se (O.S.) \\ * Correspondence: ferreira.guilherme@ufba.br (G.A.F.); lennart.piculell@fkem1.lu.se (L.P.) \\ + Current Address: Department of Physical Chemistry, Institute of Chemistry, Federal University of Bahia, \\ Salvador 40170-115, Brazil.
}

Citation: Ferreira, G.A.; Loh, W.; Topgaard, D.; Söderman, O.; Piculell, L. Molecular Assembly in Block Copolymer-Surfactant Nanoparticle Dispersions: Information on Molecular Exchange and Apparent Solubility from High-Resolution and PFG NMR. Polymers 2021, 13, 3265. https://doi.org/10.3390/ polym13193265

Academic Editors: Ksenija Kogej, Rita Dias and Per Hansson

Received: 28 August 2021

Accepted: 20 September 2021

Published: 25 September 2021

Publisher's Note: MDPI stays neutral with regard to jurisdictional claims in published maps and institutional affiliations.

Copyright: (c) 2021 by the authors. Licensee MDPI, Basel, Switzerland. This article is an open access article distributed under the terms and conditions of the Creative Commons Attribution (CC BY) license (https:/ / creativecommons.org/licenses/by/ $4.0 /)$.

\begin{abstract}
Internally structured block copolymer-surfactant particles are formed when the complex salts of ionic-neutral block copolymers neutralized by surfactant counterions are dispersed in aqueous media. Here, we report the ${ }^{1} \mathrm{H}$ NMR signal intensities and self-diffusion coefficients $(D$, from pulsed field gradient nuclear magnetic resonance, PFG NMR) of trimethyl alkylammonium surfactant ions and the poly(acrylamide)-block-poly(acrylate) (PAAm-b-PA) polyions forming such particles. The results reveal the presence of an "NMR-invisible" (slowly exchanging) fraction of aggregated surfactant ions in the particle core and an "NMR-visible" fraction consisting of surface surfactant ions in rapid exchange with the surfactant ions dissociated into the aqueous domain. They also confirm that the neutral PAAm blocks are exposed to water at the particle surface, while the PA blocks are buried in the particle core. The self-diffusion of the polyions closely agree with the self-diffusion of a hydrophobic probe molecule solubilized in the particles, showing that essentially all copolymer chains are incorporated in the aggregates. Through centrifugation, we prepared macroscopically phase-separated systems with a phase concentrated in particles separated from a clear dilute phase. $D$ values for the surfactant and block copolymer indicated that the dilute phase contained small aggregates (ca. $5 \mathrm{~nm}$ ) of surfactant ions and a few anionic-neutral block copolymer chains. Regardless of the overall concentration of the sample, the fraction of block copolymer found in the dilute phase was nearly constant. This indicates that the dilute fraction represented a tail of small particles created by the dispersion process rather than a true thermodynamic solubility of the complex salts.
\end{abstract}

Keywords: block copolymer-surfactant complexes; molecular exchange; diffusion NMR

\section{Introduction}

Several reports have studied the electrostatic complexation between water-soluble neutral-ionic block copolymers (BCP) and oppositely charged species in solution. The resulting structures are collectively known in the literature as complex coacervate core micelles, C3Ms, featuring a hydrated core and containing the complexed oppositely charged units and a stabilizer shell of neutral water-soluble polymeric blocks [1-6].

The most commonly studied C3Ms contain block-copolymers and surfactants. In recent years, stoichiometric (with respect to charge) mixtures of poly(acrylamide)-blockpoly(acrylate) (PAAm- $b$-PA) and a cationic surfactant have been extensively studied, where the samples were obtained by simply mixing surfactant and BCP stock solutions also containing the "original" simple counterions. The results showed that the resulting aggregates had an average diameter of $50 \mathrm{~nm}$ and contained surfactant-rich cores showing no long-range order [7-10]. 
Recently, studies of the dispersions of the above-mentioned type of complexes have been extended by employing an alternative methodology based on the complex salt (CS) approach [11]. In the latter approach, stoichiometric block copolymer complex salts (BCPCS) free of simple ions are prepared by simply neutralizing the acrylic acid units of the $\mathrm{BCP}$ with the hydroxide salt of the alkyltrimethylammonium cationic surfactant.

By dispersing the freeze-dried BCPCS in water, particles with an average diameter of approximately $300 \mathrm{~nm}$ with cores displaying long-range liquid crystalline structures are obtained, as shown in studies by our group in recent years [12-15]. The colloidal properties and the core structures were studied for particles of varying composition, including additionally added components, such as cosurfactants.

Recently, phase behavior as a function of the water content was investigated in concentrated aqueous BCPCS systems [16]. The latter study showed that the BCPCS did not truly dissolve in water. Mixtures containing more than approximately $50 \mathrm{wt} \%$ water phaseseparated into a concentrated ordered phase and a dilute phase. Because of the similar densities of the BCPCS and water, a separation into two macroscopic phases was difficult to achieve in normal water, even after extensive centrifugation where a uniformly turbid mixture was obtained. However, when $\mathrm{D}_{2} \mathrm{O}$ was used as a solvent, the centrifugation of water-rich samples resulted in the separation of a dilute bottom phase from a concentrated top phase. DLS results revealed the presence of small (between approximately 10 and $30 \mathrm{~nm}$ ) particles in the optically clear dilute phases, but no further information on the nature or composition of these particles was obtained.

Regardless of the composition of the BCPCS or the concentration of their dispersions in water, our knowledge on the molecular aggregation of C3Ms is incomplete. Methods such as scattering techniques and electron microscopy are primarily sensitive to the large particles that are formed, but information on the possible presence of small particles and dissociated individual molecules-including the dynamics of molecular exchange processes-is still lacking.

In the present work, we therefore use high-resolution and pulsed field gradient (PFG) ${ }^{1} \mathrm{H}$ NMR to study the molecular assembly in BCPCS dispersions. High-resolution ${ }^{1} \mathrm{H}$ NMR spectra inform us of the molecular composition of the particles and of the molecular exchange dynamics. PFG NMR methods measure the molecular translational diffusion [17] and have been employed in a variety of surfactants and BCP systems [18-23] to estimate the size of their molecular assemblies via the Stokes-Einstein equation. Moreover, information on the possible presence of bound and dissociated surfactant and BCP species can be obtained via their average diffusion coefficients.

\section{Materials and Methods}

Chemicals: The synthesis of the block copolymer PAAm $133-b-\mathrm{PAA}_{49}$ was described earlier [12], while PAAm $422-b-\mathrm{PAA}_{69}$ was a gift from Rhodia (Cranbury, NJ, USA). The subscripts refer to the weight average number of repeating units of each block (Table 1). Both polymers have been used in previous studies by our group, and their characterization is described in references $[13,16]$. Dodecyl- and hexadecyltrimethylammonium bromide; $\mathrm{C}_{12} \mathrm{TABr}$ and $\mathrm{C}_{16} \mathrm{TABr}$ of $99 \%$ purity; $\mathrm{D}_{2} \mathrm{O}$ (99.9\% D atoms); and hexamethyldisiloxane, HMDSO, were purchased from Sigma-Aldrich (Saint Louis, MO, USA) and were used as received. Deionized water with a resistivity above $18.2 \mathrm{M} \Omega \cdot \mathrm{cm}^{-1}$, obtained in a Milli-Q ${ }^{\circledR}$ system, was used in the preparation of the BCPCS.

Table 1. Weight average molar mass of the blocks and dispersity (PDI) of the PAAm- $b$-PAA block copolymers (in the acid forms) employed in this work.

\begin{tabular}{cccc}
\hline Block Copolymer & PAAm $/ \mathbf{g} \cdot \mathbf{~ m o l}^{-\mathbf{1}}$ & PAA/g $\cdot \mathbf{m o l}^{\mathbf{- 1}}$ & PDI $^{\mathbf{a}}$ \\
\hline PAAm $_{133}-b-$ PAA $_{49}$ & 9415 & 3500 & $2.1^{\mathrm{b}}$ \\
PAAm $_{432}-b-\mathrm{PAA}_{70}$ & 30,680 & 5000 & $1.6^{\mathrm{c}}$ \\
\hline${ }^{\mathrm{a}}$ PDI $=\mathrm{M}_{\mathrm{w}} / \mathrm{M}_{\mathrm{n}}$, as determined by gel permeation chromatography. ${ }^{\mathrm{b}}$ According to ref. [16]. ${ }^{\mathrm{c}}$ According to ref. [10].
\end{tabular}


Preparation of the BCPCS: Three BCPCS were prepared following the general procedure described earlier [12-16]. Briefly, the hydroxide form of the surfactants, obtained by an ion-exchange step, was titrated with aqueous solutions of the BCP in the acid form until the equivalence point ( $\mathrm{pH}$ 8.6-8.9). The resulting mixtures were left overnight at $4{ }^{\circ} \mathrm{C}$, after which their $\mathrm{pH}$ values were checked and, when necessary, adjusted to the equivalence point with the corresponding BCP solution. BCPCS in the solid form were isolated from the solution by freeze-drying the mixture and were subsequently kept in a desiccator. The three BCPCS obtained by employing this methodology will be referred to as $\mathrm{C}_{12} \mathrm{~S}, \mathrm{C}_{16} \mathrm{~S}$, and $\mathrm{C}_{12} \mathrm{~L}$, respectively, where the terms $\mathrm{C}_{12}$ and $\mathrm{C}_{16}$ refer to the surfactant alkyl chain length and where $S$ and $\mathrm{L}$ refer to the length of the PAAm block in the $\mathrm{BCP}$ employed in the synthesis: $\mathrm{S}$ for the $\mathrm{BCP}$ with a short PAAm block $\left(\mathrm{PAAm}_{133}-b-\mathrm{PA}_{49}\right)$ and $\mathrm{L}$ for the $\mathrm{BCP}$ with a long PAAm block (PAAm $\left.432-b-\mathrm{PA}_{70}\right)$.

Preparation of particle dispersions: Particle dispersions at the desired concentrations were prepared by vortexing the appropriate amounts of solid BCPCS in $\mathrm{D}_{2} \mathrm{O}$ (Vortex-Genie 2 mixer-Scientific Industries, operating at $3200 \mathrm{rpm}$ ) for approximately $1 \mathrm{~min}$. The samples thus prepared will be referred to as intact dispersions. All of the intact dispersions for the high-resolution NMR spectra were prepared with a charge concentration of $25 \mathrm{mM}$ of surfactant ion and/or polyion charges. For the self-diffusion measurements, intact dispersions with the final solid concentrations of $0.1,1.0$, or $10.0 \mathrm{wt} \%$ were prepared. In addition, centrifuged dispersions were prepared by the centrifugation of $0.1,1.0$, and $10.0 \mathrm{wt} \%$ intact dispersions for $24 \mathrm{~h}$ at $1000 \mathrm{~g}$. The resulting concentrated (upper) and dilute (bottom) phases were collected and studied individually.

Preparation of particle dispersions with HMDSO: Particle dispersions labeled with the hydrophobic probe HMDSO $[24,25]$ were obtained by dispersing each of the three investigated BCPCS in $\mathrm{D}_{2} \mathrm{O}$ to achieve $1.0 \mathrm{wt} \%$ solid concentration. Pure HMDSO at a probe-to-surfactant molar ratio of 0.01 was added to each dispersion, which was then homogenized with the aid of a vortex mixer. As reference samples, $25 \mathrm{mM}$ micellar solutions of $\mathrm{C}_{12} \mathrm{TABr}$ and $\mathrm{C}_{16} \mathrm{TABr}$ were also prepared and mixed with HMDSO at a probe-to-surfactant molar ratio of 0.01 .

NMR measurements: High-resolution ${ }^{1} \mathrm{H}-\mathrm{NMR}$ spectra were obtained using a 400 $\mathrm{MHz}$ Bruker Avance spectrometer (Billerica, MA, USA) for the intact dispersions as well as for the solutions of the individual components (surfactants and block copolymers), all of which were at a charge concentration of $25 \mathrm{mM}$. The signal intensities were quantitatively compared by integrating the corresponding peak areas. The self-diffusion experiments, based on low-resolution ${ }^{1} \mathrm{H}-\mathrm{NMR}$ spectra, were performed using a Bruker Avance-II 200 (Billerica, MA, USA) operating at a $200 \mathrm{MHz}$ proton resonance frequency equipped with a Bruker diffusion probe with a maximum gradient strength of $9.6 \mathrm{Tm}^{-1}$, unless otherwise stated. The pulsed field gradients (PFGs) were generated in a Bruker DIFF-25 gradient probe driven by a GREAT-40 unit, except where otherwise indicated. These measurements were performed using a maximum gradient strength $(g)$ of $4.52 \mathrm{Tm}^{-1}$ with a pulsed gradient duration $(\delta)$ of $1.0 \mathrm{~ms}$, and the time between the start of two gradient pulses $(\Delta)$ was $20 \mathrm{~ms}$. The stimulated echo (PFG-STE) method was used, with a pulse sequence of $90^{\circ}-\tau_{1}-90^{\circ}-\tau_{2}-90^{\circ}-\tau_{1}-$ echo [26]. In the PFG-STE experiment, the attenuation of the signal intensity $I$ was given by [17]:

$$
I=I_{o} \exp \left(-D \gamma^{2} \delta^{2} g^{2}\left(\Delta-\frac{1}{3} \delta\right)\right)
$$

where $I_{o}$ is the signal intensity in the absence of gradients, $D$ is the translational diffusion coefficient, and $\gamma$ is the magnetogyric ratio. All of the measurements were conducted at $25.0 \pm 0.5^{\circ} \mathrm{C}$. All of the data processing and the fitting of the diffusion coefficients were achieved using the spectrometer software (Topspin 2.1, Bruker, Billerica, MA, USA). Each diffusion coefficient results from the fit of 12-14 data points (peak areas). The number of significant figures given for the $D$ values is based on the reproducibility of the measurements performed in duplicate for selected samples studied at the same conditions. 
In principle, any non-overlapping peak from the surfactant, the polyion, or the HMDSO probe can be used to analyze the diffusion of the respective species. Based on a two-state model, assuming that the molecule is either "bound" to the particle or "free" as a non-aggregated unimer in solution, and that the exchange between those two states is fast, the observed diffusion coefficient is given by [17]:

$$
D_{\text {obs }}=\alpha D_{\text {free }}+(1-\alpha) D_{\text {particle }}
$$

where $D_{o b s}$ is the observed diffusion coefficient of the molecule (surfactant or polyion) in the dispersion, $D_{\text {particle }}$ is the particle diffusion coefficient, $D_{\text {free }}$ is the diffusion coefficient of free surfactant ion or polyion, and $\alpha$ is the fraction of free molecules. The unimer diffusion coefficients of the non-aggregated surfactant ions and polyions, corresponding to $D_{\text {free }}$ in Equation (2), were measured in $\mathrm{D}_{2} \mathrm{O}$ and are presented in Table 2.

Table 2. Unimer self-diffusion coefficients of surfactant ions and polyions obtained using the indicated peaks in ${ }^{1} \mathrm{H}$ NMR spectra.

\begin{tabular}{ccc}
\hline Species & $\mathbf{D} / \times \mathbf{1 0}^{\mathbf{- 1 0}} \mathbf{~ m}^{\mathbf{2}} \mathbf{.} \mathbf{s}^{\mathbf{1}}$ & Peak Position/ppm \\
\hline $\mathrm{C}_{12} \mathrm{TA}^{+\mathrm{a}}$ & 4.86 & 3.1 \\
$\mathrm{C}_{16} \mathrm{TA}^{+\mathrm{b}}$ & 4.14 & 3.1 \\
PAAm $_{133}-b-\mathrm{PAA}_{49}{ }^{c}$ & 0.66 & 1.7 \\
PAAm $_{432}-b-\mathrm{PAA}_{70} \mathrm{~d}$ & 0.31 & 1.7 \\
\hline
\end{tabular}

${ }^{\mathrm{a}}$ In $10 \mathrm{mM} \mathrm{C}_{12}$ TABr solution $(\mathrm{cmc}=12 \mathrm{mM}) .{ }^{\mathrm{b}}$ In $0.7 \mathrm{mM} \mathrm{C}_{16}$ TABr solution $(\mathrm{cmc}=1 \mathrm{mM}) .{ }^{\mathrm{c}}$ In $10 \mathrm{mM}$, based on AA monomer, PAAm $_{133}-b$-PAA 49 solution. ${ }^{\mathrm{d}}$ In $10 \mathrm{mM}$, based on AA monomer, PAAm $432-b$-PAA 70 solution.

\section{Results}

\subsection{High-Resolution ${ }^{1} H$-NMR Spectra}

High-resolution ${ }^{1} \mathrm{H}$-NMR spectra were obtained for freshly prepared intact $\mathrm{C}_{12} \mathrm{~S}$ and $\mathrm{C}_{12} \mathrm{~L}$ dispersions as well as for the individual surfactant and block copolymer solutions. All of the samples have the same charge concentration of $25 \mathrm{mM}$ for the surfactant ion and/or polyion charges. Spectra from the solutions of the surfactant and the block copolymers are shown in Figure 1 together with chemical structures and peak assignments. For the surfactant (Figure 1A), the peak in the interval 3.0-3.1 ppm can be attributed to the protons of the trimethyl $\left(\left(-\mathrm{CH}_{3}\right)_{3}\right)$ group in the surfactant headgroup, whereas the peaks at 3.3, $1.7-1.8$, and $1.2-1.3 \mathrm{ppm}$ can be attributed to the methylene $\left(-\mathrm{CH}_{2}-\right)$ groups of the surfactant alkyl chain. The peak at around $0.8 \mathrm{ppm}$ corresponds to the terminal methyl $\left(-\mathrm{CH}_{3}\right)$ group of the surfactant chain.

Peak assignments for the block copolymers are based on published spectra for the homopolymers PAA [27] and PAAm [28]. The two methine $(-\mathrm{CH}<)$ groups of the copolymer blocks (Figure 1B) give rise to one partly resolved peak just above $2.3 \mathrm{ppm}$ for PA, which is only visible for PAAm $133-b-\mathrm{PAA}_{49}$, and two peaks at 2.25 and $2.1 \mathrm{ppm}$ for PAAm. The methylene $\left(-\mathrm{CH}_{2}-\right)$ groups of the two blocks give rise to three peaks for each polymer repeat unit, one resolved peak at $1.85 \mathrm{ppm}$ from PA, two overlapping peaks from both repeat units at 1.7 and $1.55 \mathrm{ppm}$, and a shoulder at $1.45 \mathrm{ppm}$ from PAAm. The relative contributions to the peaks at 1.7 and $1.55 \mathrm{ppm}$ differ between PA and PAAm: the peak at 1.7 is the most intense for PA, whereas the $1.55 \mathrm{ppm}$ peak is the most intense for PAAm. 

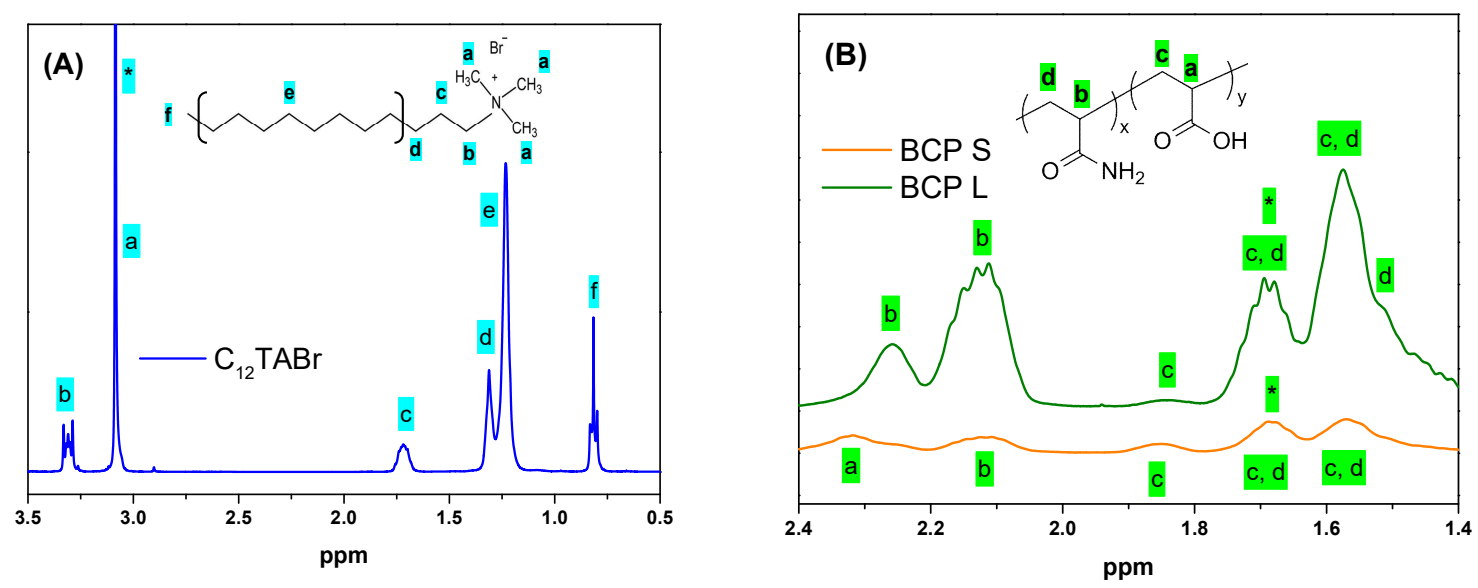

Figure 1. ${ }^{1} \mathrm{H}$ NMR spectra for (A) surfactant and (B) block copolymer solutions with labeled peaks according to the chemical structures presented in the inset. The peaks used for diffusion experiments are indicated with asterisks. The terms $\mathrm{S}$ and $\mathrm{L}$ refer to the length of the PAAm block in the BCP (short and long, respectively). Solutions contain the individual components (surfactants and block copolymers) at a charge concentration of $25 \mathrm{mM}$, which is above the cmc for the surfactant. Note in (B) that since the PA concentration was the nearly the same for the two block copolymer solutions, the intensities of all of the PAAm peaks are much stronger for PAAm $432-b-\mathrm{PAA}_{70}(\mathrm{BCP} \mathrm{L})$ than they are for PAAm $133-b-\mathrm{PAA} 49$ (BCP S).

The obtained spectra for $\mathrm{C}_{12} \mathrm{~S}$ and for $\mathrm{C}_{12} \mathrm{~L}$ are displayed at different expansions in the three panels of Figure 2 to highlight the peaks and chemical shifts related to surfactant or the block copolymer, respectively. Each of the panels, A, B and C, also includes a spectrum of the reference surfactant or the block copolymer solution, which were recorded under exactly the same conditions as the BCPCS sample and were plotted at the same magnification along the $y$ axis to allow a direct comparison. Figure 2A shows that the surfactant peaks are broader and are much less intense in the dispersions than in the reference surfactant solution, especially for $\mathrm{C}_{12} \mathrm{~S}$. For the block copolymer, the resolved peaks from PA at 2.3 and 1.85 ppm disappear in the dispersions, while the resolved peaks from PAAm (2.25 and $2.1 \mathrm{ppm})$ and the overlapping peaks from PA and PAAm are less affected. The disappearance of the PA signals in the $\mathrm{C}_{12} \mathrm{~S}$ spectrum was confirmed in a separate experiment. A single experiment (not shown) on $\mathrm{C}_{16} \mathrm{~S}$ showed a partial but not total loss of the PA signal intensities compared to the PAAm signal intensities. Figure 2B,C show that there is a contribution from the surfactant to the peak at $1.7 \mathrm{ppm}$ in both dispersions but, nevertheless, the composite signals from the dispersions are much less intense than the signal from the surfactant alone at 1.7 ppm (see Figure 2A).


Figure 2. ${ }^{1} \mathrm{H}$ NMR spectra for intact dispersions compared to spectra for surfactant and block copolymer individual solutions recorded under the same conditions and at the same concentrations as in the dispersions. (A) shows comparisons of the surfactant peaks while $(B, C)$ compare the block copolymer peaks. The term $C_{12}$ denotes the surfactant alkyl chain length whereas $\mathrm{S}$ or L refer to the length of the PAAm block in the BCP (short or long, respectively).

\subsection{Compositions of Phase-Separated Samples}

Macroscopically phase-separated dispersions of $\mathrm{C}_{12} \mathrm{~S}, \mathrm{C}_{16} \mathrm{~S}$, and $\mathrm{C}_{12} \mathrm{~L}$ were prepared by means of the centrifugation of the intact dispersions prepared at $0.1,1.0$, and $10.0 \mathrm{wt} \%$, as 
described in the Experimental section. In each case, centrifugation resulted in the creation of a clear, dilute bottom phase separated from a concentrated, turbid top phase. The latter phase corresponded to approximately $10 \%$ of the sample volume, irrespective of the initial BCPCS concentration. PFG NMR measurements were performed on the separated dilute and concentrated phases as well as on the corresponding intact dispersions. The low-resolution ${ }^{1} \mathrm{H}$ NMR spectra obtained in the PFG experiments were also analyzed. The spectra from the dilute phases were quite similar to those from the intact dispersions although, naturally, the intensities in the dilute phases were much weaker. For sensitivity reasons, the integrals from the composite (see above) methylene peak at $1.7 \mathrm{ppm}$ were used as a measure of the BCPCS concentration. Figure 3 shows that for all three of the investigated samples, the BCPCS concentration in the dilute phase was, to a good approximation, proportional to the that of the intact dispersions. For an estimate of the absolute concentrations in the dilute phases, the peak integrals were compared to a calibration curve based on the spectra for the short polymer at different concentrations. From this comparison, we conclude that in all of the dilute phases, the concentration of BCP was in the range $5-10 \%$ of the concentration in the intact dispersion. Note that, due to intensity losses (see Section 3.1 above), the spectra from the intact dispersions were not suitable as a reference to measure BCPCS concentration. However, since the dilute phases contained only small particles (see below), it was assumed that their spectra did not suffer any intensity loss.
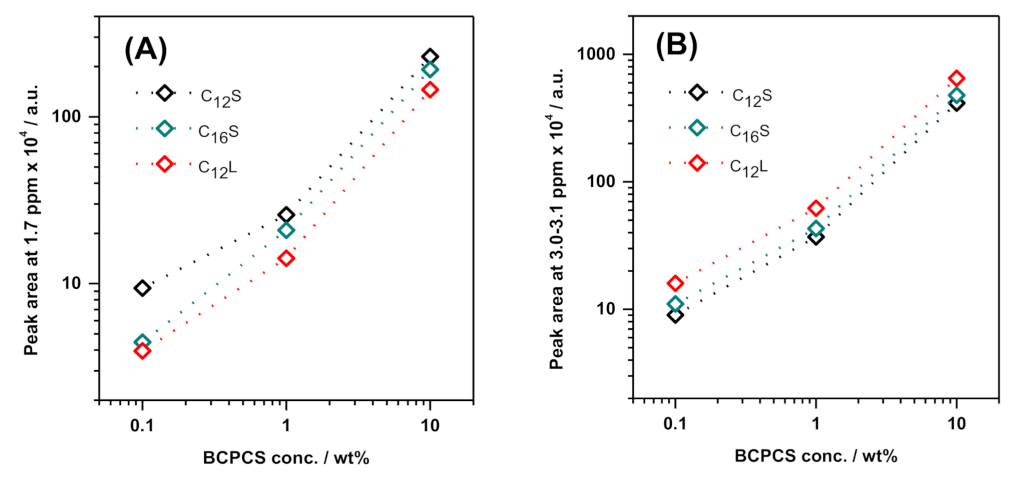

Figure 3. Variation of peak areas at (A) $1.7 \mathrm{ppm}$ (composite peak associated with the polyion) and (B) 3.0-3.1 ppm (surfactant peak) in the dilute phase obtained in the centrifuged samples as a function of overall initial BCPCS concentration. The lines are guides to the eye. Note the log-log scale. The terms $C_{12}$ and $C_{16}$ denote the surfactant alkyl chain length, and $S$ and $L$ refer to the length of the PAAm block in the BCP (short and long, respectively).

\subsection{Self-Diffusion Measurements}

Self-diffusion measurements were performed on both the intact and phase-separated (centrifuged) samples. The surfactant ion self-diffusion was analyzed from the methyl proton signal of the trimethylammonium headgroup in the ${ }^{1} \mathrm{H}$ NMR spectra (peak at $3.1 \mathrm{ppm}-$ Figure 1A). The BCP diffusion coefficient was obtained from the methylene group signal at $1.7 \mathrm{ppm}$, which, again, was for sensitivity reasons (Figure 1B). Figure 4 shows the obtained self-diffusion coefficients for the ionic species in all of the investigated intact samples.

In a control experiment, freshly prepared intact samples at $1 \mathrm{wt} \%$ solid concentration were labeled with the hydrophobic probe HMDSO at a probe-to-surfactant molar ratio of 0.01 in order to reach approximately one probe molecule per micelle, a protocol used in previous studies of similarly labelled micellar surfactant solutions [23,24]. A comparison of the low-resolution ${ }^{1} \mathrm{H}$ NMR spectra obtained for the HMDSO-labeled dispersions and the corresponding surfactant solutions (Figure S1) confirms that the surfactant signal intensities are significantly reduced in the dispersions. Table 3 shows the obtained selfdiffusion coefficient values for the surfactant ions, polyions, and hydrophobic probe for the three investigated particle dispersions. For each sample, the HMDSO and polyion diffusion coefficients differ by less than $10 \%$. 


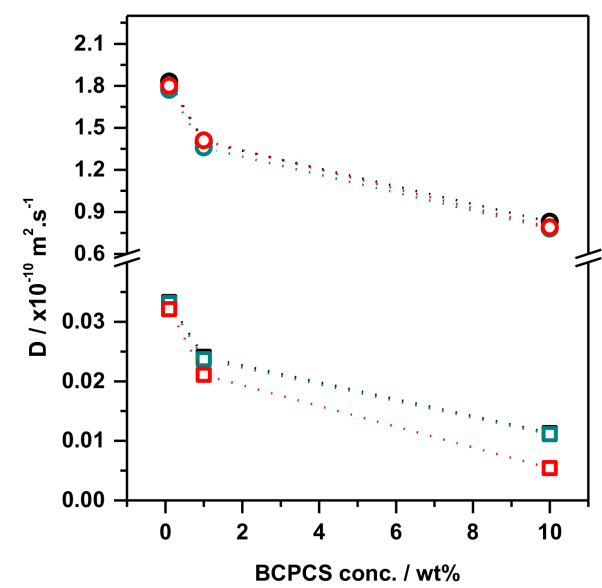

Figure 4. Self-diffusion coefficient values $(D)$ as a function of BCPCS concentration in intact samples. Symbols denote the $\mathrm{C}_{12} \mathrm{TA}^{+}$ion $(\bigcirc)$ and polyion $(\square)$ in $\mathrm{C}_{12} \mathrm{~S}$; the $\mathrm{C}_{16} \mathrm{TA}^{+}$ion $(\bigcirc)$ and polyion $(\square)$ in $\mathrm{C}_{16} \mathrm{~S}$; and the $\mathrm{C}_{12} \mathrm{TA}^{+}$ion $(\bigcirc)$ and polyion $(\square)$ in $\mathrm{C}_{12} \mathrm{~L}$. The lines are guides for the eye. The terms $\mathrm{C}_{12}$ and $C_{16}$ refer to the surfactant alkyl chain length, whereas $S$ and $L$ refer to the length of the PAAm block in the BCP (short and long, respectively).

Table 3. Self-diffusion coefficients (D) for surfactant ions, polyions, and HMDSO in intact samples at $1 \mathrm{wt} \%$ BCPCS.

\begin{tabular}{ccc}
\hline BCPCS & Species & D/m $^{\mathbf{2} . \mathbf{s}^{-1}}$ \\
\hline \multirow{3}{*}{$\mathrm{C}_{12} \mathrm{~S}$} & $\mathrm{C}_{12} \mathrm{TA}^{+}$ & $1.43 \times 10^{-10}$ \\
& $\mathrm{PAAm}_{133}-b-\mathrm{PA}_{49}$ & $2.33 \times 10^{-12}$ \\
& $\mathrm{HMDSO}_{12}$ & $2.12 \times 10^{-12}$ \\
$\mathrm{C}_{12} \mathrm{~L}$ & $\mathrm{C}_{12} \mathrm{TA}^{+}$ & $1.38 \times 10^{-10}$ \\
& $\mathrm{PAAm}_{432}-b-\mathrm{PA}_{70}$ & $2.10 \times 10^{-12}$ \\
& $\mathrm{HMDSO}^{\mathrm{C}}$ & $1.90 \times 10^{-12}$ \\
& $\mathrm{C}_{16} \mathrm{TA}^{+}$ & $1.32 \times 10^{-10}$ \\
& $\mathrm{PAAm}_{133}-b-\mathrm{PA}_{49}$ & $2.35 \times 10^{-12}$ \\
& $\mathrm{HMDSO}$ & $2.18 \times 10^{-12}$ \\
\hline
\end{tabular}

The self-diffusion coefficients of the surfactant ions and polyions measured in the concentrated and dilute phases of the centrifuged samples obtained from BCPCS dispersions at different overall concentrations are displayed in Figure 5.
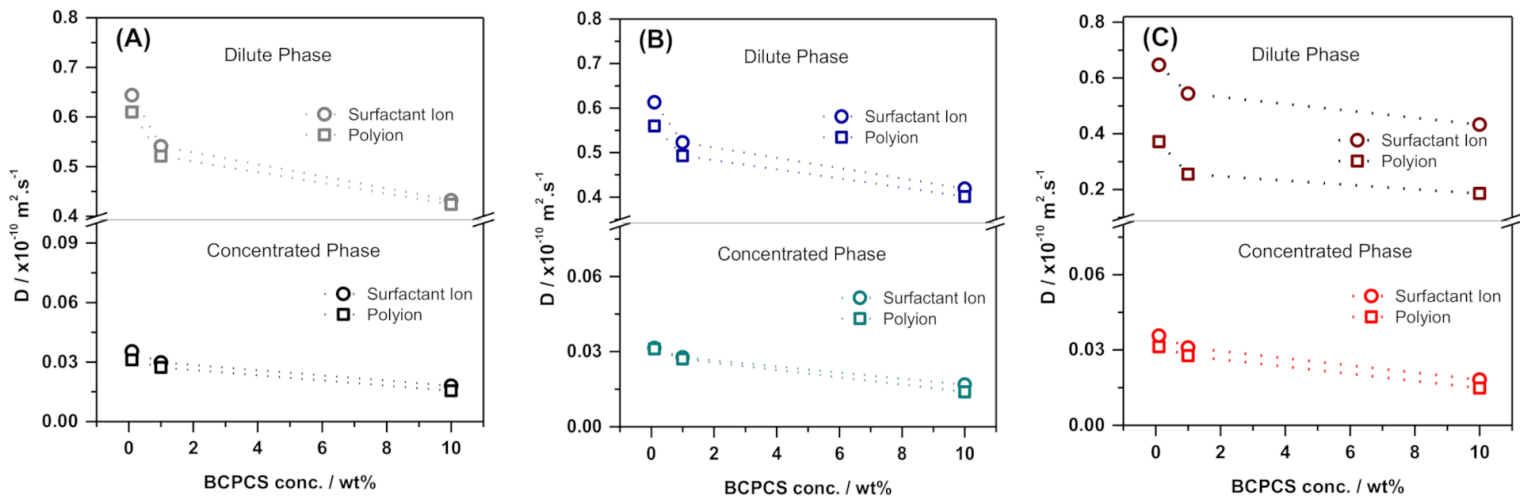

Figure 5. Self-diffusion coefficients (D) for surfactant ions and polyions in the concentrated and dilute phases obtained for centrifuged samples at different (overall) initial concentrations for $(A) C_{12} S,(B) C_{16} S$, and $(C) C_{12} L$. The lines are guides for the eye. The terms $C_{12}$ and $C_{16}$ refer to the surfactant alkyl chain length, whereas $S$ and $L$ refer to the length of the PAAm block in the BCP (short and long, respectively). 


\section{Discussion}

\subsection{High-Resolution ${ }^{1} H$ NMR Spectra}

High-resolution ${ }^{1} \mathrm{H}$ NMR spectra (Figure 2) obtained for intact BCPCS samples at $1.0 \mathrm{wt} \%$ display characteristic signals belonging to both surfactant and block copolymers. A significant loss of signal intensity is consistently seen for all of the surfactant peaks compared to the pure surfactant solution (Figure 2A), which indicates that only a portion of the surfactant content is seen in the spectra and contributes to the measured self-diffusion coefficients. The spectra obtained for the micellar solutions and the particle dispersions labeled with HMDSO also confirm a substantial loss of signal intensity for the surfactant peaks relative to HMDSO (Figure S1). This indicates that the surfactant molecules in the particles fall into two categories, as illustrated in Figure 6.

(A)

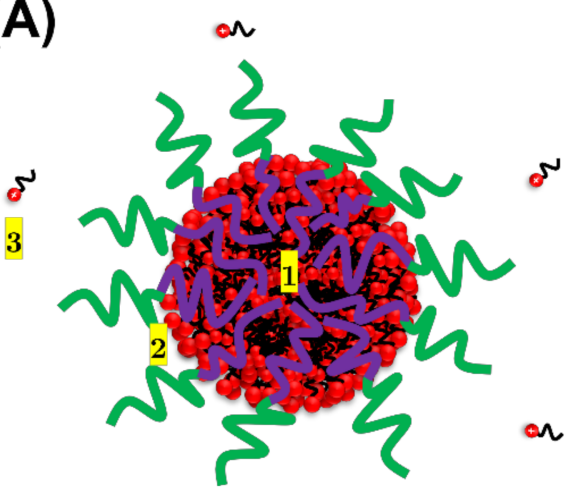

(B)



Figure 6. Schematic illustration of distinguishable populations of surfactant ions in BCPCS particle dispersions: (A) large particles (predominating in intact samples and concentrated phases) with a dense liquid crystalline core formed by surfactant micelles surrounded by the polyion chains; (B) small aggregates (predominating in dilute phases) formed by aggregated surfactant ions neutralized by a few polyion chains. In (A), the sites labeled as 1, 2, and 3 correspond to the core, surface, and free (dissociated) surfactant ions, respectively. The exchange between the core and surface surfactant ions is slow, whereas the exchange between the surface and free surfactant ions is fast. In (B), the sites labeled as 4 and 5 correspond to the surface and free surfactant ions, which undergo a fast exchange. In both cases, the aggregate shape is arbitrarily depicted as spherical. Green, purple, red, and black segments denote the PAAm block, the PA block, the surfactant headgroup, and the surfactant alkyl chain, respectively.

The "NMR visible" surfactant ions consist of "free" surfactant unimers, which are dissociated into the water domain, and "surface" surfactant ions, which are situated sufficiently close to the particle-water interface in order to undergo rapid exchange with the free surfactant ions. The timescale for rapid exchange is milliseconds, which was determined by the PFG experiment. The "NMR invisible" surfactant ions, by contrast, are surfactant molecules in the interior of the particles, which exchange too slowly with the surface and free fractions to contribute to a visible high-resolution signal. Instead, the invisible fraction gives rise to a separate signal, which is too broad to be detected in the high-resolution NMR experiment. The cores of the large BCPCS particles are known to contain surfactant ion aggregates arranged in ordered liquid crystalline structures, resulting in slow surfactant diffusion coefficients and very broad spectra [19]. The ${ }^{1} \mathrm{H}$ NMR linewidth is determined by molecular dynamics that average the dipolar spin-spin coupling to zero. As explained in more detail in reference [19], the reorientation of the surfactant aggregates in the concentrated liquid crystalline structure of the particle core is severely hindered, and the relatively rapid diffusion of the surfactant ions over the surfaces of the surfactant aggregates does not suffice to average the ${ }^{1} \mathrm{H}-{ }^{1} \mathrm{H}$ dipolar coupling to zero since the aggregates are anisometric. The diffusional exchange of surfactant ions between the aggregates of the uncorrelated orientations is also slow. 
Regarding the block copolymer signals (Figure 2B,C), the well-resolved signals from PAAm are only moderately affected by incorporation in the particles, which is consistent with the a priori notion that the PAAm blocks are all situated at the surface of the particle in a hydrated shell, where they experience considerable motional freedom. By contrast, the high-resolution PA signals from the particles have disappeared entirely for $\mathrm{C}_{12} \mathrm{~S}$ (Figure $2 \mathrm{~B}, \mathrm{C}$ ) and partially for $\mathrm{C}_{16} \mathrm{~S}$. This suggests that the PA chains are largely buried in the particle core, where they reorient much more slowly and anisotropically, giving rise to very broad signals, similar to the NMR-invisible surfactant ions in the core.

\subsection{Self-Diffusion Measurements}

The self-diffusion coefficients for polyions in intact samples (Figure 4) and in the concentrated phase of the centrifuged samples (Figure 5A-C) were similar, indicating that they predominantly contained particles of similar sizes. The close similarity (Table 3 ) between the polyion diffusion and the diffusion of HMDSO, which was quantitatively incorporated in the surfactant micelles inside the BCPCS particles, confirms that the polyion diffusion essentially measures the particle diffusion.

A striking feature seen in Figure 4 is the very rapid surfactant diffusion in intact dispersions. For the $1 \mathrm{wt} \%$ dispersions of the three BCPCS that were investigated, the measured surfactant diffusion was approximately one third of the unimer diffusion measured in aqueous surfactant solutions below the $\mathrm{cmc}$ (Table 2). At this stage, we are unable to quantitatively explain such a rapid surfactant diffusion, which was reproducibly observed by measurements of several preparations, which also used different spectrometers. To qualitatively understand this result, we recall from the previous Section 4.1 that only a fraction of the surfactant ions is NMR visible, and it is only for this pool of surfactant ions that the fast exchange condition, resulting in Equation (2) above, applies. The rapidly exchanging surfactant ions must reside on the particle surfaces, but we have no independent information on the surface-to-volume ratio in the polydisperse particle dispersions. Conductivity measurements (see Supporting Information for details) show, however, that the concentration of free surfactant ions is very low (approximately $1 \mathrm{mM}$ for $1 \mathrm{wt} \% \mathrm{C}_{12} \mathrm{~S}$ or $\mathrm{C}_{16} \mathrm{~S}$ ) in the intact particle dispersions (Figure S2).

A further complicating factor in the study of the intact particle dispersions is our finding that a slow creaming process occurs for BCPCS dispersions in $\mathrm{D}_{2} \mathrm{O}$. The density difference between the particles and $\mathrm{D}_{2} \mathrm{O}$, which allows particles to be separated by centrifugation, also leads to a continuous net transport of preferentially larger particles towards the top of an intact sample under gravity. We could observe the creaming process by monitoring the NMR signal intensities and the self-diffusion coefficients for the samples left unstirred in the spectrometer over $50 \mathrm{~h}$, as reported in detail in the Supplementary Materials (see Figures S3 and S4 and the accompanying text). The signal intensity decreased with time, indicating a decreasing concentration of BCPCS particles in the lower part of the NMR tube, which was probed in the NMR experiment. Simultaneously, the recorded average particle diffusion increased with time, indicating that the remaining particles had a smaller average size compared to the full distribution. Significant creaming already occurred at the timescale of hours. However, a gentle mixing of an aged sample after 3 days restored the properties of a fresh dispersion (Table S2 and Figure S5), confirming that creaming, rather than changes in the particle size distribution as such, was indeed the mechanism responsible for the time-dependent phenomena.

One may ask to what extent the particular NMR features found here for surfactant ions in intact dispersions, namely the reduced proton signal intensity and the apparent rapid diffusion, are dependent on the complex salt approach used to produce the dispersions. To check this, we made additional measurements on dispersions of $\mathrm{C}_{12} \mathrm{TABr}$ with the sodium salts of the two $\mathrm{BCP}$ prepared by the conventional mixing protocol in stoichiometric proportions. The conventionally prepared dispersions indeed displayed the same features as the intact dispersions: a much-decreased surfactant signal intensity (Figure S6) coupled with a very rapid surfactant ion diffusion coefficient (Table 4). Table 4 also shows that the 
polyion diffusion, which corresponds to the particle diffusion, was significantly more rapid for the conventionally prepared particles, corresponding to a smaller hydrodynamic radius of approximately $50 \mathrm{~nm}$. The difference in size between the differently prepared particles was confirmed by DLS measurements (Table 4) and was in line with previous data from scattering and microscopy techniques for similarly prepared particle dispersions [7-10].

Table 4. Self-diffusion coefficients for surfactant ions $\left(D_{\text {surf }}\right)$ and polyions $\left(D_{\text {pol }}\right)$ in dispersions at $25 \mathrm{mM}$ of charge concentration: comparing dispersions prepared by the complex salt approach to those prepared by a conventional protocol. The table also includes hydrodynamic radii $\left(\mathrm{R}_{\mathrm{H}}\right)$ obtained from dynamic light scattering (DLS) and from the polyion self-diffusion coefficients using the Stokes-Einstein relation. Self-diffusion measurements performed in a $400 \mathrm{MHz}$ Bruker Avance spectrometer.

\begin{tabular}{|c|c|c|c|c|}
\hline Sample & $D_{\text {surf }} \times 10^{-10} / \mathrm{m}^{2} \cdot \mathrm{s}^{-1}$ & $D_{p o l} \times 10^{-12} / \mathrm{m}^{2} \cdot \mathrm{s}^{-1}$ & $R_{H}(\mathrm{DLS}) / \mathrm{nm}^{\mathrm{c}}$ & $R_{H}\left(D_{p o l}\right) / \mathrm{nm}^{\mathrm{d}}$ \\
\hline $\mathrm{C}_{12} \mathrm{~S}^{\mathrm{a}}$ & 1.60 & 3.00 & 120 & 131 \\
\hline $\mathrm{C}_{12} \mathrm{~L}^{\mathrm{a}}$ & 1.60 & 2.40 & 130 & 164 \\
\hline $\mathrm{C}_{12} \mathrm{~S}^{\mathrm{b}}$ & 1.40 & 7.00 & 50 & 56 \\
\hline $\mathrm{C}_{12} \mathrm{~L}^{\mathrm{b}}$ & 1.70 & 6.30 & 55 & 63 \\
\hline
\end{tabular}

a Samples prepared by the complex salt approach, as described in Experimental section. ${ }^{\mathrm{b}}$ Samples of $\mathrm{C}_{12} \mathrm{TABr}$ with the sodium salts of the two BCP prepared by conventional mixing in stoichiometric proportions. ${ }^{c}$ Determined by dynamic light scattering measurements performed as in ref. [13]. ${ }^{\mathrm{d}}$ Calculated from the polyion self-diffusion coefficient using the Stokes-Einstein equation (see refs. [22,23]).

We will now turn to the centrifuged dispersions of all three BCPCS. In the separated concentrated phases of all three BCPCS, the observed self-diffusion coefficients for the polyion and surfactant ion were quite similar, with a surfactant ion diffusion that was only slightly more rapid than the polyion ion diffusion. This result supports the notion that surfactant ions are essentially quantitatively incorporated in large BCPCS particles. Our main objective when studying centrifuged samples was, however, to obtain more detailed information on the small particles remaining in the dilute phases of these samples.

Clearly, for a truly binary mixture of water with a BCPCS containing strictly monodisperse polyions and surfactant counterions, the concentration of BCPCS detected in the dilute phase would be independent of the overall concentration of the system at thermodynamic equilibrium since it would then simply correspond to the solubility of the BCPCS in water. One conceivable origin of a constant soluble polymer fraction in the dilute phase could thus be the existence of a molecularly distinct fraction of polymer that is unable to form complexes with the surfactant due to a low or vanishing content of charged units. However, this explanation is ruled out in our case by the finding from NMR that the dilute phase also contains surfactant ions and that the diffusion coefficients of these surfactant ions show that they are largely associated with the polyions. The surfactant ion diffusion coefficients are much lower than the respective unimer diffusion coefficients (Table 2), and their concentration dependence closely follows that of the polyions (Figure 4).

The polyion diffusion in the dilute phases, in turn, varies, as expected from the molecular masses of the respective polyions; that is, it increases in the sequence $D\left(\mathrm{C}_{12} \mathrm{~L}\right)$ $<D\left(\mathrm{C}_{16} \mathrm{~S}\right) \approx D\left(\mathrm{C}_{12} \mathrm{~S}\right)$. For all of the particles the polyion diffusion coefficients show a rather strong concentration dependence, and for the lowest initial concentration, they, in fact, approach the diffusion coefficients of the respective single polyions (compare data in Figure 4 and Table 2). Collectively, the data thus indicate that the dilute systems contain small BCPCS complexes, each containing aggregated surfactant ions and at most a few polyions, the latter being of a size that is comparable to the average size of the $\mathrm{BCP}$ used in the complex formation (Figure 6).

In conclusion, the properties of the polyions in the dilute phase complexes do not seem to differ significantly from the average properties of the respective polyion samples. We must therefore consider a different explanation for the existence of a constant fraction of apparently soluble BCPCS particles. We suggest that the small aggregates in the dilute phase simply represent a fraction of very small aggregates from a wide distribution of aggregate sizes. We must then assume that a reproducible size distribution is created during 
the dispersion of the BCPCS in water, that the distribution is only weakly dependent on the initial concentration of BCPCS, and that the created particles are stable in time. The last conclusion is supported by the fact that the original NMR intensities and average diffusion coefficients were obtained in samples subjected to three days of creaming after a gentle mixing of the samples. (Figure S5 and Table S1).

In any case, the complexes of the dilute phases are sufficiently small enough to justify the assumption of rapid exchange and narrow NMR linewidths, so the use of Equation (2) should be legitimate for these systems. Hence, the equation has been used to calculate the dissociated fraction of the surfactant ions $(\alpha)$, and the results are shown in Figure 7.

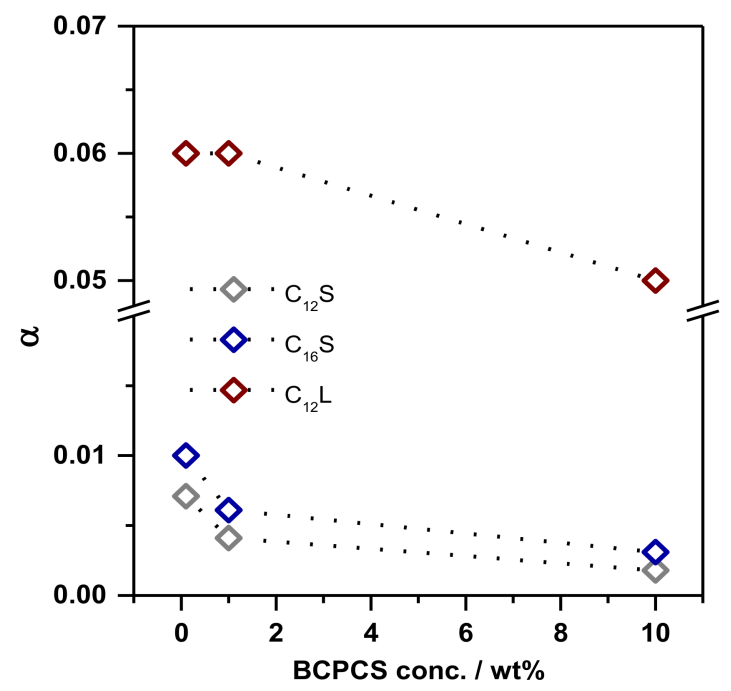

Figure 7. Fraction of dissociated surfactant ions $(\alpha)$ as a function of BCPCS concentration in the dilute phases obtained by centrifuging BCPCS samples at different overall (initial) concentrations. The lines are guides for the eye. The terms $C_{12}$ and $C_{16}$ refer to the surfactant alkyl chain length, and $S$ and $L$ refer to the length of the PAAm block in the BCP (short and long, respectively).

In the calculations, we assumed that $D_{\text {particle }}$ equals the measured diffusion coefficient of the polyion in each sample. For $\mathrm{C}_{12} \mathrm{~S}$ and $\mathrm{C}_{16} \mathrm{~S}$, the fractions of the free surfactant ions are very small, confirming that the latter are in fact self-assembled into micelles complexed to the polyions, as sketched in Figure 6B, which is in agreement with experimental evidence on the nature of polyion-surfactant ion complexation in dilute solution $[5,6]$. Interestingly, $\alpha$ is significantly larger for $\mathrm{C} 12 \mathrm{~L}$, indicating that the presence of a large neutral block on the polyion may partially hinder the micellization of the surfactant at the polyion.

\section{Conclusions}

The dispersion of BCPCS in water by vortexing generates a rather wide but stable and reproducible size distribution of BCPCS particles. When $\mathrm{D}_{2} \mathrm{O}$ rather than $\mathrm{H}_{2} \mathrm{O}$ is used as the solvent, the particles slowly separate according to size by creaming.

In particular, the distribution includes a fraction of small aggregates that do not separate out when the dispersion is subjected to centrifugation but remain in solution as one or a few (depending on concentration) block copolymer chains attached to one or a few surfactant ion micelles. The concentration of such small aggregates in the dilute phase increases in proportion to the overall content of BCPCS, but their molecular components, the BCP in particular, do not differ measurably in composition from the bulk average. No evidence is thus found for a true thermodynamic solubility of BCPCS; the apparent solubility in the dilute phase results from the formation of small kinetically stable particles.

The much larger BCPCS particles that dominate the full distribution contain a surface pool of surfactant ions in rapid exchange with a small fraction of surfactant ions that have dissociated from the particles, separated from a slowly exchanging pool of surfactant ions inside the particle cores. This picture holds also for particles that have been conventionally 
prepared by mixing surfactant solutions with block polyion, including their respective simple counterions.

The neutral PAAm blocks are highly mobile, indicating that they extend out into the aqueous phase. By contrast, the polyacrylate blocks are largely buried in the particle cores. The fraction of block polyions dissociated from the particles is essentially zero.

Supplementary Materials: The following are available online at https:/ /www.mdpi.com/article/10 $.3390 /$ polym13193265/s1, Figure S1: Low-resolution ${ }^{1} \mathrm{H}$ NMR spectra for samples labeled with the hydrophobic probe HMDSO. (A) micellar solutions of $\mathrm{C}_{12} \mathrm{TABr}$ and $\mathrm{C}_{16} \mathrm{TABr}$ at $25 \mathrm{mM}$. (B) BCPCS intact dispersions at $1.0 \mathrm{wt} \%$. In all cases, the surfactant-to-HMDS molar ratio is 0.01 . For both cases, * denotes the peak used to measure the self-diffusion of HMDSO, Figure S2: (A). Conductivity ( $\mathrm{k}$ ) of $\mathrm{C}_{12} \mathrm{TABr}$ solutions as a function of $\mathrm{C}_{12} \mathrm{TA}^{+}$surfactant ion concentration (open symbols). The horizontal lines denote the conductivity of the intact samples of $\mathrm{C}_{12} \mathrm{~S}$ (red) and $\mathrm{C}_{12} \mathrm{~L}$ (blue) at $1 \mathrm{wt} \%$. (B). Conductivity ( $k$ ) of the $\mathrm{C}_{16} \mathrm{TABr}$ solutions as a function of the $\mathrm{C}_{16} \mathrm{TA}^{+}$surfactant ion concentration (open symbols). The horizontal green line denotes the conductivity of the intact $\mathrm{C}_{16} \mathrm{~S}$ sample at $1 \mathrm{wt} \%$, Figure S3: Self-diffusion coefficients (D) for surfactant ion (circles) and polyion (squares) in an intact $1.0 \mathrm{wt}$. $\% \mathrm{C}_{12} \mathrm{~S}$ as a function of time for a sample prepared in $\mathrm{D}_{2} \mathrm{O}$. Red points refer to a sample in which the solvent is a 30:70 $\mathrm{H}_{2} \mathrm{O}: \mathrm{D}_{2} \mathrm{O}$ mixture, Figure S4: Low-resolution ${ }^{1} \mathrm{H} N M R$ spectra for an intact $1.0 \mathrm{wt}$. \% $\mathrm{C}_{12} \mathrm{~S}$ sample as a function of time of sample preparation in (A) $\mathrm{D}_{2} \mathrm{O}$; (B) 30:70 $\mathrm{H}_{2} \mathrm{O}: \mathrm{D}_{2} \mathrm{O}$ mixture, Figure S5: Low-resolution ${ }^{1} \mathrm{H}$ NMR spectra for a $1.0 \mathrm{wt}$. \% intact $\mathrm{C}_{12} \mathrm{~S}$ dispersion 3 days after sample preparation followed by redispersion in (A) $\mathrm{D}_{2} \mathrm{O}$; (B) 30:70 $\mathrm{H}_{2} \mathrm{O}: \mathrm{D}_{2} \mathrm{O}$ mixture. For comparison purposes, spectra obtained at $0 \mathrm{~h}$ and $50 \mathrm{~h}$ are presented again for both samples, Figure S6: High-resolution ${ }^{1} \mathrm{H}$ NMR spectra obtained for $\mathrm{C}_{12} \mathrm{~S}$ and $\mathrm{C}_{12} \mathrm{~L}$ nanoparticle dispersions prepared by the conventional protocol of mixing of the aqueous solutions of surfactant salt and sodium salt of BCP at a charge ratio of 1 . The molar charge concentration for each species is equal to $25 \mathrm{mM}$, Table S1: Self-diffusion coefficients obtained for surfactant ion $\left(D_{\text {surf }}\right)$ and polyion $\left(D_{\text {pol }}\right)$ in a $1 \mathrm{wt} \%$ intact $\mathrm{C}_{12} \mathrm{~S}$ sample prepared in $\mathrm{D}_{2} \mathrm{O}$ and in a mixture of $\mathrm{H}_{2} \mathrm{O}: \mathrm{D}_{2} \mathrm{O}$ at different time intervals (t).

Author Contributions: Conceptualization, G.A.F., W.L. and L.P.; methodology, G.A.F.; formal analysis, G.A.F., W.L., D.T., O.S. and L.P.; writing—original draft preparation, G.A.F.; writing—review and editing, G.A.F., W.L., D.T., O.S. and L.P.; supervision, W.L. and L.P.; project administration, W.L. and L.P.; funding acquisition, W.L. and L.P. All authors have read and agreed to the published version of the manuscript.

Funding: This study was financed in part by the Coordenação de Aperfeiçoamento de Pessoal de Nível Superior-Brasil (CAPES)—Finance Code 001. FAPESP has sponsored this work (Proc. No. 2015/25406-5).

Data Availability Statement: Not applicable.

Acknowledgments: Göran Carlström (Lund University) and Suelen Gauna Trindade (UNICAMP) are acknowledged for their help with the NMR measurements. The authors thank Leticia Vitorazi for the synthesis and characterization of one of the block copolymers used in this work. G.A.F. thanks the Brazilian Agency CAPES for the PhD fellowship, and W.L. thanks CNPq for the senior researcher grant.

Conflicts of Interest: Authors declare no conflicts of interest.

\section{References}

1. Harada, H.; Kataoka, K. Formation of Polyion Complex Micelles in an Aqueous Milieu from a Pair of Oppositely Charged Block Copolymers with Poly(ethylene glycol) Segments. Macromolecules 1995, 28, 5294-5299. [CrossRef]

2. van der Burgh, S.; de Keizer, A.; Cohen Stuart, M.A. Complex Coacervation Core Micelles. Colloidal Stability and Aggregation Mechanism. Langmuir 2004, 20, 1073-1084. [CrossRef]

3. Cohen Stuart, M.A.; Hofs, B.; Voets, I.K.; de Keizer, A. Assembly of polyelectrolyte-containing block copolymers in aqueous media. Curr. Opin. Colloid Interface Sci. 2005, 10, 30-36. [CrossRef]

4. Voets, I.K.; de Keizer, A.; de Waard, P.; Frederik, P.M.; Bomans, P.H.H.; Schmalz, H.; Walther, A.; King, S.M.; Leermarkes, F.A.M.; Cohen Stuart, M.A. Double-Faced Micelles from Water-Soluble Polymers. Angew. Chem. Int. Ed. 2006, 45, 6673-6676. [CrossRef]

5. Voets, I.K.; de Keizer, A.; Cohen Stuart, M.A. Complex coacervate core micelles. Adv. Colloid Interface Sci. 2009, 147-148, 300-318. [CrossRef] 
6. Ferreira, G.A.; Loh, W. Liquid crystalline nanoparticles formed by surfactant-polyelectrolyte complexes. Curr. Opin. Colloid. Int. Sci. 2017, 32, 11-22. [CrossRef]

7. Berret, J.F.; Cristobal, G.; Hervé, P.; Grillo, I. Structure of colloidal complexes obtained from neutral/poly-electrolyte copolymers and oppositely charged surfactants. Eur. Phys. J. E 2002, 9, 301-311. [CrossRef] [PubMed]

8. Berret, J.F.; Hervé, P.; Aguerre-Chariol, O.; Oberdisse, J. Colloidal complexes obtained from charged block copolymers and surfactants: A comparison between small-angle neutron scattering, Cryo-TEM, and simulations. J. Phys. Chem. B 2003, 107, 8111-8118. [CrossRef]

9. Hervé, P.; Destarac, M.; Berret, J.-F.; Lal, J.; Oberdisse, J.; Grillo, I. Novel Core-shell Structure for Colloids Made of Neutral/Polyelectrolyte Diblock Copolymers and Oppositely Charged Surfactants. Europhys. Lett. 2002, 58, 912-918. [CrossRef]

10. Berret, J.-F.; Vigolo, B.; Eng, R.; Hervé, P.; Grillo, I.; Yang, L. Electrostatic Self-assembly of Oppositely Charged Copolymers and Surfactants: A Light, Neutron, and X-ray Scattering Study. Macromolecules 2004, 37, 4922-4930. [CrossRef]

11. Svensson, A.; Piculell, L.; Cabane, B.; Iketi, P. A New Approach to the Phase Behavior of Oppositely Charged Polymers and Surfactants. J. Phys. Chem. B 2002, 106, 1013-1018. [CrossRef]

12. Vitorazi, L.; Berret, J.-F.; Loh, W. Self-Assembly of Complex Salts of Cationic Surfactants and Anionic-Neutral Block Copolymers. Dispersions with Liquid-Crystalline Internal Structure. Langmuir 2013, 29, 14024-14033. [CrossRef]

13. Ferreira, G.A.; Loh, W. Addition of n-Alcohols Induces a Variety of Liquid-Crystalline Structures in Surfactant-Rich Cores of Dispersed Block Copolymer/Surfactant Nanoparticles. ACS Omega 2016, 1, 1104-1113. [CrossRef] [PubMed]

14. Carneiro, N.M.; Percebom, A.M.; Loh, W. Quest for Thermoresponsive Block Copolymer Nanoparticles with Liquid-Crystalline Surfactant Cores. ACS Omega 2017, 2, 5518-5528. [CrossRef] [PubMed]

15. Ferreira, G.A.; Loh, W. Planet-Satellite Nanostructures Based on Block Copolymer-Surfactant Nanoparticles Surface-Decorated with Gold and Silver: A New Strategy for Interfacial Catalysis. Adv. Mat. Interfaces 2019, 13, 1900348. [CrossRef]

16. Ferreira, G.A.; Piculell, L.; Loh, W. Hydration-dependent hierarchical structures in block copolymer-surfactant complex salts. Macromolecules 2018, 51, 9915-9924. [CrossRef]

17. Price, W.S. NMR Studies of Translational Motion; Cambridge University Press: Cambridge, UK, 2009.

18. Söderman, O.; Stilbs, S. NMR Studies of Complex Surfactant Systems. Prog. Nucl. Magn. Reson. Spectrosc. 1994, 26, 445-482. [CrossRef]

19. Svensson, A.; Topgaard, D.; Piculell, L.; Söderman, O. Molecular Self-Diffusion in Micellar and Discrete Cubic Phases of an Ionic Surfactant with Mixed Monovalent/Polymeric Counterions. J. Phys. Chem. B 2003, 107, 13241-13250. [CrossRef]

20. Cabaleiro-Lago, C.; Nilsson, M.; Söderman, O. Self-Diffusion NMR Studies of the Host-Guest Interaction between $\beta$-Cyclodextrin and Alkyltrimethylammonium Bromide Surfactants. Langmuir 2005, 21, 11637-11644. [CrossRef]

21. Bernardes, J.S.; da Silva, M.A.; Piculell, L.; Loh, W. Reverse micelles with spines: L2 phases of surfactant ion-polyion complex salts, n-alcohols and water investigated by rheology, NMR diffusion and SAXS measurements. Soft Matter 2010, 6, 144-153. [CrossRef]

22. Percebom, A.M.; Janiak, J.; Schillén, K.; Piculell, L.; Loh, W. Micellization of water-soluble complex salts of an ionic surfactant with hairy polymeric counterions. Soft Matter 2013, 9, 515-526. [CrossRef]

23. Janiak, J.; Piculell, L.; Schillén, K.; Lundberg, D. Responsive release of polyanions from soluble aggregates formed with a hydrolyzable cationic surfactant and a nonionic surfactant. Soft Matter 2013, 9, 4103-4112. [CrossRef]

24. Jansson, M.; Stilbs, P. A Comparative Study of Organic Counterion Binding to Micelles with the Fourier Transform NMR Self-Diffusion Technique. J. Phys. Chem. 1985, 89, 4868-4873. [CrossRef]

25. Ginley, M.; Henriksson, U. Self-diffusion study of counterion complexation in aqueous micellar lithium dodecyl sulfate solutions. J. Colloid. Interface Sci. 1992, 150, 281-284. [CrossRef]

26. Tanner, J.E. Use of stimulated echo in NMR diffusion studies. J. Chem. Phys. 1970, 52, 2523-2526. [CrossRef]

27. Spěváček, J.; Suchopárek, M.; Al-Alawi, S. Characterization of the stereochemical structure of poly(acrylic acid) by one- and two-dimensional ${ }^{13} \mathrm{C}-{ }^{1} \mathrm{H}$ nuclear magnetic resonance spectra. Polymer 1995, 36, 4125-4130. [CrossRef]

28. Hikichi, K.; Ikura, M.; Yasuda, M. Two-Dimensional ${ }^{1} \mathrm{H}$ and ${ }^{13} \mathrm{C}$ Nuclear Magnetic Resonance Studies of Poly(acrylamide). Polym. J. 1988, 20, 851-859. [CrossRef] 